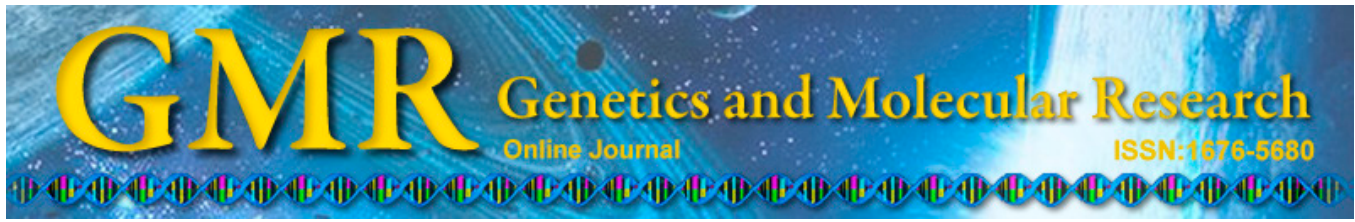

\title{
Significance of sarcomere gene mutation in patients with dilated cardiomyopathy
}

\author{
Y.D. Li ${ }^{1 *}$, Y.T. Ji ${ }^{1 *}$, X.H. Zhou', H.L. Li' ${ }^{2}$ H.T. Zhang ${ }^{3}$, Y. Zhang', \\ J.X. Li ${ }^{1}$, Q. Xing ${ }^{1}$, J.H. Zhang ${ }^{1}$, Y.F. Hong ${ }^{1}$ and B.P. Tang ${ }^{1}$ \\ ${ }^{1}$ Pacing and Electrophysiology Department, \\ The First Affiliated Hospital of Xinjiang Medical University, \\ Urumuqi, Xinjiang, China \\ ${ }^{2}$ Heart Rhythm Institute and Endocrinology, \\ University of Oklahoma Health Sciences Center, Oklahoma City, OK \\ ${ }^{3}$ Cardiology Department, People's Hospital of Burqin County, \\ Urumqi, Xinjiang, China \\ *Theses authors contributed equally to this study. \\ Corresponding author: B.P. Tang \\ E-mail: tangbaopeng@hotmail.com
}

Genet. Mol. Res. 14 (3): 11200-11210 (2015)

Received January 22, 2015

Accepted May 8, 2015

Published September 22, 2015

DOI http://dx.doi.org/10.4238/2015.September.22.14

\begin{abstract}
Dilated cardiomyopathy (DCM) is a myocardial disease with a high mortality rate. Approximately 40 genes have been found to be associated with DCM to date. Non-familial DCM can also be caused by gene mutations, suggesting that genetic factors were involved in the pathogenesis of DCM; therefore genetic testing is beneficial for the early diagnosis of DCM, which can facilitate the implementation of preventive measures by and within patient's families. Here, we investigated the underlying genetic mutations involved in the cause of patients with DCM. This prospective study included 240 patients with idiopathic DCM and 240 healthy volunteers. Subject clinical data were collected and polymerase chain reaction amplification was carried out on subject DNA for three candidate genes tropomyosin (TPMI), cardiac troponin $\mathrm{T}$ type-2 (TNNT2), and nuclear lamina protein $\mathrm{A} / \mathrm{C}$.
\end{abstract}


Single nucleotide polymorphism (SNP) loci were detected in the TPM1 (rs1071646) and TNNT2 (rs3729547) genes, respectively. The genotype distributions and allele frequencies were found to satisfy Hardy-Weinberg equilibrium, which indicated that the group was representative. Statistically significant differences were found between the variant frequencies in the two SNP loci between the Kazakh patients with idiopathic DCM (IDCM) and healthy volunteers. A significant difference in the genotype distributions $(\mathrm{P}=0.000)$ and allele frequencies $(\mathrm{P}=0.000)$ of SNP rs1071646, and another significant difference in the genotype distributions $(\mathrm{P}=0.000)$ and allele frequencies $(\mathrm{P}=$ 0.039 ) of SNP rs3729547 between Kazakhs with IDCM and Kazakh controls. These results suggest that the TPM1 (rs1071646) and TNNT2 (rs3729547) gene variants might represent risk factors for patients with DCM in the Kazakh population.

Key words: Dilated cardiomyopathy; Cardiac troponin T type-2 gene; Gene mutation; Single nucleotide polymorphism; Tropomyosin gene; Nuclear lamina protein gene

\section{INTRODUCTION}

Dilated cardiomyopathy (DCM) is a primary myocardial disease with high morbidity and mortality rates in children and adults. Its primary clinical features include the left ventricle or ventricular systolic and diastolic dysfunction, which might result in complications such as chronic heart failure, arrhythmia, sudden death, and cardiovascular diseases. The incident rate of DCM is approximately 30-40 per one lakh persons. Furthermore, the incidence is higher in males than in female, and is 2.5 times higher in blacks than in the white population (Burkett and Hershberger, 2005). Idiopathic DCM (IDCM) is diagnosed if the incidence of DCM is isolated with only a single member of a family involved, and if the cause of the disease is unclear. If at least two closely related family members are diagnosed with sporadic DCM or a young family member were reported to have died of sudden cardiac death, then familial DCM (FDCM) is indicated (Burkett and Hershberger, 2005). To date, 40 genes have been reported to be associated with DCM (Fatkin et al., 2010). Sometimes variety of genetic mutations together leads to the occurrence of DCM. At the same time, the study found that DCM in patients with familial transmissibility is up to $30-50 \%$, and the risk of disease is known to be increased in the families of patients with DCM; therefore early genetic screening should be performed in these families. Furthermore, genetic factors play an important role in the pathogenesis of DCM (Hershberger et al., 2009) as well as in the risk. Therefore, understanding the genetic mechanism and genetic testing are not only beneficial for the early diagnosis of DCM, but also can prevent disease in the patient families through implementation of prophylactic measure.

Most genes associated with the onset of DCM exhibit standard patterns of autosomal dominant, autosomal recessive, and X-linked inheritance (Mestroni et al., 1999). These genes are involved in the encoding of myocardial skeleton, nuclear membrane, cardiac muscle, sarcomere, and mitochondrial proteins (Mogensen et al., 2004). This spectrum of genes is different from that found to underlie hypertrophic cardiomyopathy (HCM) (Richard et al., 2003); 
However in 2000, Kamisago et al. (2000) found that the genes, encoding the sarcomere and cytoskeleton were not only associated with HCM, but also with the onset of DCM. Accordingly, genes encoding of tropomyosin (TPM1), myocardial actin, troponin T (TNNT2), troponin I and $\mathrm{C}$, myosin-binding protein, and connectin have subsequently been demonstrated to be associated with IDCM (Møller et al., 2009). In addition, lamin A/C (LMNA) and X-linked muscular dystrophy proteins were also found to be involved in DCM occurrence and their disruption was shown to lead to heart blockage and bone atrophy (Schönberger and Seidman, 2001).

However, the role of these genes in IDCM remains unclear and further study is needed to clarify this issue. In this study, we investigated potential mutational changes of three of the known candidate genes: TPM1, TNNT2, and $L M N A$ along with their associations with patients with IDCM of Kazakh and Han descent in Xinjiang, China.

\section{MATERIAL AND METHODS}

\section{Ethical approval}

This study was reviewed and approved by the Ethical Committee of the First Affiliated Hospital to Xinjiang Medical University, and all participants provided signed written informed consent before study enrollment.

\section{Subjects}

This prospective study was conducted at the Pacing and Electrophysiology Department, First Affiliated Hospital to Xinjiang Medical University, Urumqi, Xinjiang, China. Patients with IDCM enrolled in this study included: 120 Kazakh (48 men and 72 women) and 120 Han (62 men and 58women) ethnic individuals. The control group consisted of 240 healthy volunteers including 120 Kazakh (56 men and 64 women) and 120 Han (67 men and 53 women) individuals.

The study inclusion criteria were as follows: 1) Patient left ventricular end diastolic dimension (LVEDD) greater than 50 and $55 \mathrm{~mm}$ for women and men, respectively; 2) left ventricular ejection fraction (LVEF) and left ventricular decurtation rate less than 45 and 25\%, respectively; and 3) expanded cardiac development revealed by X-ray and cardiothoracic ratio results greater than $50 \%$. Clinically, the diagnosis was primary based on echocardiography, X-ray chest radiography, and cardiac isotope, and cardiac computed tomography. Magnetic resonance imaging examination was of diagnostic significance for patients with cardiac localized hypertrophy. The study exclusion criteria included myocardial damage caused by other diseases such as hypertension, coronary, pulmonary, valvular, and congenital heart diseases, alcoholism and tachycardiomyopathy, pericardial, systemic, and neuromuscular diseases as per the "Guidelines for the diagnosis and management of FDCM" (Fatkin and members of the CSANZ, 2011).

\section{Clinical information}

Clinical data included the following: date of birth, gender, nationality, clinical diagnosis, age at diagnosis, family history of cardiovascular disease (myocardial infarction, hypertension, and myocarditis); toxin/drug exposure duration as well as cardiac structure and function 
including maximum left ventricular wall thickness, ejection fraction, and area (Lakdawala et al., 2012). All subjects had detailed clinical information including general information, medical history, 12-lead synchronous electrocardiogram, echocardiography, and six-minute walk test, etc.

\section{Genetic analysis}

A 2-mL sample of peripheral blood was collected and DNA was extracted from each subject. Polymerase chain reaction (PCR, China Shanghai Meiji Biological Testing Company) amplification was carried out to determine the exonic sequences of LMNA (NM_001257374.2), TPM1 (NM_000366.5), and TNNT2 (NM_000364.3), and the amplification products were purified. All gene exon sequences underwent sequencing in both the positive and negative directions followed by analysis using the DNAMAN software, and the results were compared with the standard template sequences using NCBI BLAST and the CHROMAS software to identify the gene mutation loci (amplification and sequencing were accomplished by China Shanghai Meiji Biological Testing Company). Later, the mutation loci were examined with reverse sequencing and re-sequencing for confirmation (Tables 1-3).

\begin{tabular}{|c|c|c|c|c|}
\hline Exon & Forward primer $\left(5^{\prime}-3^{\prime}\right)$ & Reverse primer $\left(5^{\prime}-3^{\prime}\right)$ & Amplicon size (bp) & Annealing temperature $\left({ }^{\circ} \mathrm{C}\right)$ \\
\hline 1 & ATC TAC GAA TCC ACG GTC & CCC AGC TCA TTG AGT TCA C & 1639 & 53 \\
\hline 2 & TAT TCG CAG GAG TCT GGT CTG A & AAA CGG CAA AGG TCA CCC & 1419 & 55 \\
\hline 3 & GGA ATA GGT GGA CGG AAC & GGT AAT AAG GGT CGA CGA & 1674 & 53 \\
\hline 4 & AGA ACC GTC ATA CTT TGG & CAC CGA TAG GAC TTT GTC & 1016 & 50 \\
\hline 5,6 & ACG ATG ACT AAC CGA AGG A & AAG GAA ACC GAA CAG AGG & 1805 & 53 \\
\hline 7,8 & ACG ATG ACT AAC CGA AGG A & GAT GTA GTG TCT TCC CTA A & 1628 & 50 \\
\hline 9 & CTG AGG TTG TCG TTG TAA & GTA GGT GTT CTA TGG TCG AT & 813 & 53 \\
\hline
\end{tabular}

Table 2. Primer sequences for TNNT2 gene amplification.

\begin{tabular}{lllcr}
\hline Exon & Forward primer $\left(5^{\prime}-3^{\prime}\right)$ & Reverse primer $\left(5^{\prime}-3^{\prime}\right)$ & Amplicon size $(\mathrm{bp})$ & Annealing temperature $\left({ }^{\circ} \mathrm{C}\right)$ \\
\hline $1,2,3$ & GTT GAA GCA AGG AAC GGG & TTT TGG TAT TGG TGT TAC GGT A & 2001 & 52 \\
$4,5,6$ & AAA CTG ACA GCC GAT GGA & CCC TAT CCC TCG TAA AGG & 2068 & 53 \\
7,8 & GCC AAA GTA ACA AAC CGA & ATG ACC TAC GAG GAA CTF AC & 1518 & 52 \\
9,10 & TGT TGA CCC TCT GTA GTC C & GAG TTG ACC GTG AGT ATA AC & 1746 & 53 \\
$11,12,13$ & AGA TTT CGT AGT TCC GT & GGG TGT ATG TGG GTT CCA & 1990 & 51 \\
14,15 & GAAACT CGT CAT CGT AGG G & GAA CTG GGA GGT AAG GAAA & 1699 & 53 \\
\hline
\end{tabular}

Table 3. Primer sequences for $L M N A$ gene amplification.

\begin{tabular}{lllrr}
\hline Exon & Forward primer $\left(5^{\prime}-3^{\prime}\right)$ & Reverse primer $\left(5^{\prime}-3^{\prime}\right)$ & Amplicon size $(\mathrm{bp})$ & Annealing temperature $\left({ }^{\circ} \mathrm{C}\right)$ \\
\hline 1 & GGG AAAG TCA GGG AAA GAT GA & GCT ATT GAA ACG GTA TCA GAG & 1536 & 53 \\
2 & ATG AGT GAA GGG AAA CCG & GAA TAG GAT TAA ACC GAC G & 739 & 50 \\
$3,4,5$ & CTC CTA CAA ACT CGT CCC & CTC TTC CCA GCG TCC TAC & 1695 & 52 \\
6,7 & GGT GTA GGA CGC TGG GAA & TCG TTC TAT GTG GGT TCT CG & 1883 & 52 \\
$8,9,10$ & CCA CAT AGA ACG AGT TTC CG & GAC CAT TCC TTC CCT CAC C & 1111 & 55 \\
$11,12,13$ & ACC AAA CGG ATG ACC CAC & CTA CCC TTA CTC CAC CCT & 2424 & 55 \\
\hline
\end{tabular}




\section{Statistical analysis}

The SPSS 17.0 software (SPSS, Chicago, IL, USA) was used for statistical analysis. Clinical data are reported as means \pm standard deviation. A Hardy-Weinberg equilibrium test was used to test the population representativeness of the sample. A direct counting method was applied to calculate the genotype distributions and allele frequencies. Chi-square $\left(\chi^{2}\right)$ test was used to analyze the differences of genotype distributions and allele frequencies between groups.

\section{RESULTS}

\section{Clinical information}

Statistical analyses on the clinical data of the 240 patients with IDCM and the 240 healthy volunteers were conducted. The distributions of gender and age between the patients with IDCM and the control groups showed no statistical differences. The differences of LVEDD and LVEF between the two groups were statistically significant (Table 4). A HardyWeinberg equilibrium test was used to assess the TPM1 and TNNT2 gene variants, which indicated that the group was representative of the population (Tables 5 and 6).

Table 4. Baseline information.
\begin{tabular}{llccc} 
\\
\hline Ethnicity & Data & Patients & Controls & P \\
\hline Kazakh & Gender (M/F) & 48772 & $56 / 64$ & $0.297^{\#}$ \\
& Age (years) & $46.5 \pm 11.89$ & $48.7 \pm 6.65$ & 0.616 \\
& LVEF (\%) & $37.2 \pm 4.04$ & $50.8 \pm 4.75$ & 0.000 \\
LVEDD (mm) & $56.4 \pm 6.41$ & $47.9 \pm 6.69$ & 0.001 \\
& Gender (M/F) & $62 / 58$ & $67 / 53$ & $0.517^{\#}$ \\
& Age (years) & $51.6 \pm 9.17$ & $59.1 \pm 7.41$ & 0.060 \\
& LVEF (\%) & $39.1 \pm 2.84$ & $45.2 \pm 3.04$ & 0.000 \\
& LVEDD (mm) & $59.9 \pm 6.80$ & $50.5 \pm 3.24$ & 0.001 \\
\hline
\end{tabular}

\#Does not meet chi-square test conditions; continuity correction was used instead. LVEF = left ventricular ejection fraction; LVEDD = left ventricular end diastolic dimension. This table presents baseline information, including gender, age, LVEF, and LVEDD. The distributions of gender and age between the patients with IDCM and the control groups were not statistically significant. The differences of LVEDD and LVEF between the two groups were statistically significant. Gender, LVEF and LVEDD were represented by the mean and standard deviation, detected by analysis of variance. Age detected by the chi square test.

Table 5. Test of Hardy-Weinberg equilibrium for TPM1 genotypes.
\begin{tabular}{lccccc}
\hline Group & \multicolumn{7}{c}{ rs1071646 genotype } \\
\cline { 2 - 5 } & $\mathrm{C} / \mathrm{C}$ & $\mathrm{C} / \mathrm{A}$ & $1 / \mathrm{A}$ & $\chi^{2}$ & Prob exact test \\
\hline Patients (K) & $0(0)$ & $4(0.03)$ & $116(0.97)$ & 0.034 & 0.852 \\
Controls (K) & $0(0)$ & $24(0.20)$ & $96(0.80)$ & 1.481 & 0.223 \\
Patients (H) & $36(0.3)$ & $56(0.47)$ & $28(0.23)$ & 0.468 & 0.493 \\
Controls (H) & $20(0.17)$ & $48(0.40)$ & $52(0.43)$ & 2.310 & 0.128 \\
\hline P
\end{tabular}

$\mathrm{P}>0.05$ indicates a representative group; $\mathrm{K}=$ Kazakh; $\mathrm{H}=\mathrm{Han}$; Prob $=$ probability. The parentheses represented the percentage of genotype. 
Table 6. Test of Hardy-Weinberg equilibrium for TNNT2 genotypes.

\begin{tabular}{lccccc}
\hline Group & \multicolumn{5}{c}{ rs3729547 genotype } \\
\cline { 2 - 6 } & $\mathrm{C} / \mathrm{C}$ & $\mathrm{C} / \mathrm{T}$ & $\mathrm{T} / \mathrm{T}$ & $\chi^{2}$ & Prob Exact test \\
\hline Patients (K) & $0(0)$ & $12(0.05)$ & $108(0.95)$ & 0.332 & 0.564 \\
Controls (K) & $0(0)$ & $25(0.21)$ & $95(0.79)$ & 1.622 & 0.202 \\
Patients (H) & $24(0.20)$ & $52(0.43)$ & $44(0.37)$ & 1.414 & 0.234 \\
Controls (H) & $26(0.22)$ & $65(0.54)$ & $29(0.24)$ & 0.840 & 0.357 \\
\hline P &
\end{tabular}

$\mathrm{P}>0.05$ indicates a representative group; $\mathrm{K}=$ Kazakh; $\mathrm{H}=\mathrm{Han}$; Prob $=$ probability. The parentheses represented the percentage of genotype.

\section{Gene sequencing results}

PCR amplification was carried on three candidate genes, and the amplification products were sequenced and a total of two mutation loci were identified: The two identified mutations, TPM1 (rs1071646, Ala151Ala) and TNNT2 (rs3729547, Ile116Ile), led to base replacement and were therefore considered pathological, their potential to lead to DCM was clarified using Pubmed. The genotype distributions and allele frequencies at the TPM1 and TNNT2 mutation loci between patients with IDCM of Chinese Xinjiang Kazakh ethnicity and the healthy population showed statistically significant differences. Figure 1 shows the rs 1071646 locus in the TPM1 gene. Figure 2 shows the rs3729547 locus in the TNNT2 gene. In the Han Chinese population in Xinjiang, there were no statistical differences for the two candidate genes between the two groups. No mutation loci were identified for $L M N A$ between the patients with IDCM and the healthy population (Tables 7 and 8).
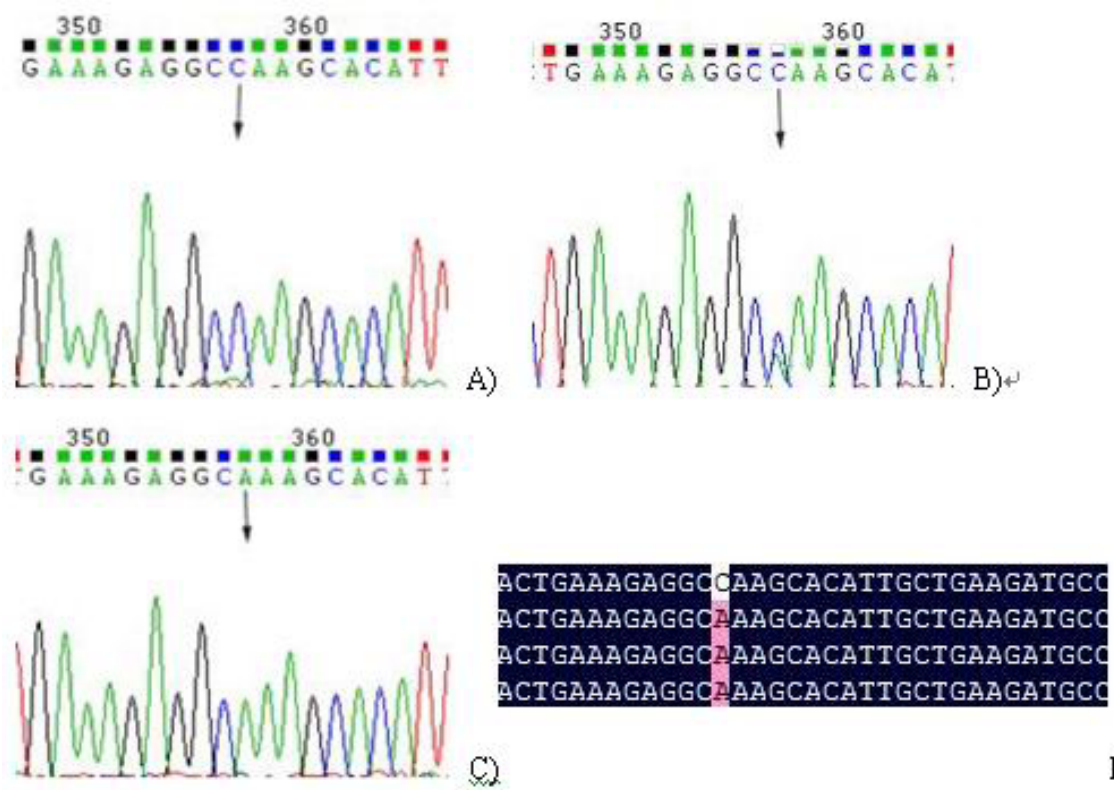

C)

D)

Figure 1. TPM1 exon $4 \mathrm{C}>\mathrm{A}$ Ala151Ala mutation. A. Wild-type homozygote C/C. B. Mutant heterozygote C/A. C. Wild-type homozygote A/A. D. Comparison of results using DNAMAN. Here, the top sequence represents the wild-type sequence. 

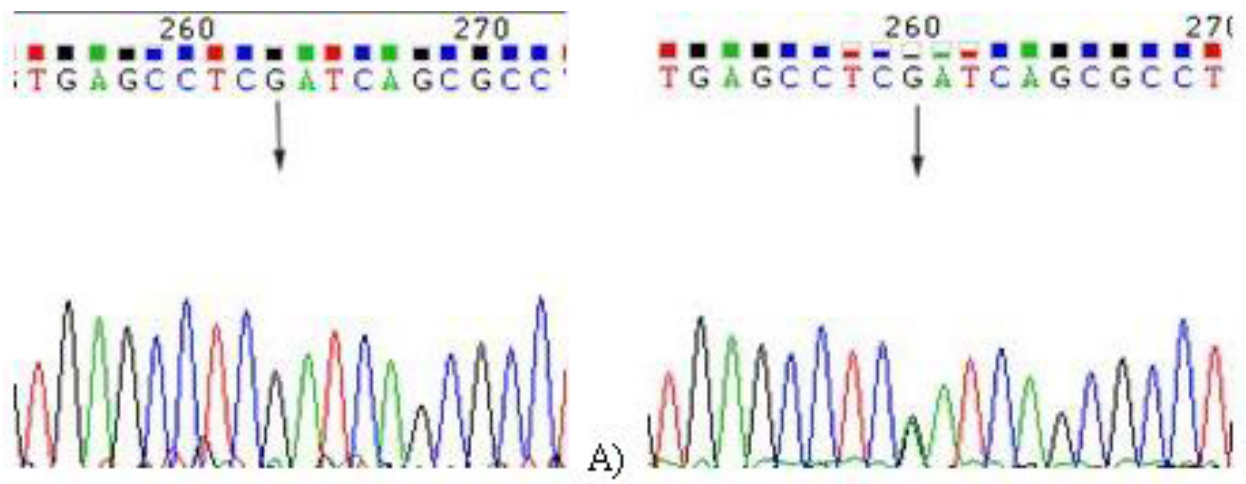

A)
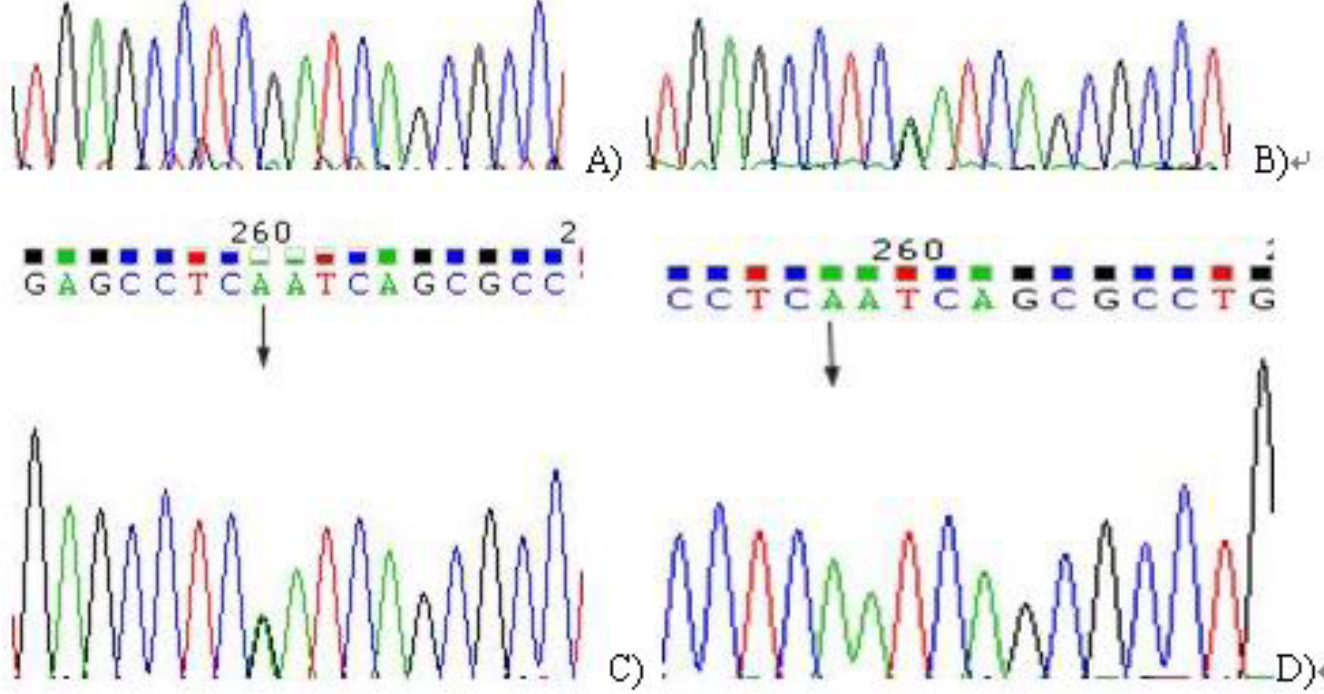

C)!

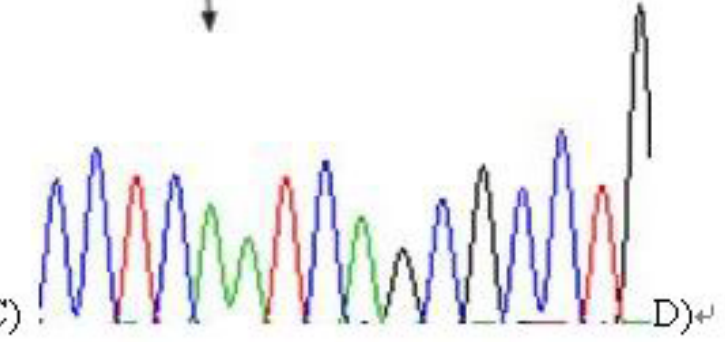

\section{EAAAGTGAGCCTOGATCAGCGCCTGCAACTCATT \\ BAAAGTGAGCCTCZATCAGCGCCTGCAACTCATT \\ CAAAGTGAGCCTOZATCAGCGCCTGCAACTCATT \\ EAAAGTGAGCCTOZATCAGCGCCTGCAACTCATT}

E)

Figure 2. TNNT2 exon 10 complementary sequence G > A Ile116Ile. A. Wild-type homozygote G/G. B. and C. Mutant heterozygote G/A. D. Mutant homozygous A/A. E. Comparison of results using DNAMAN. Here, the top sequence represents the wide-type sequence.

Table 7. Chi-square tests for the TPMI gene polymorphism.

\begin{tabular}{|c|c|c|c|c|c|c|c|c|c|c|}
\hline \multirow[t]{2}{*}{ Group } & \multicolumn{5}{|c|}{ rs1071646 genotype } & \multicolumn{4}{|c|}{ rs 1071646 allele } & \multirow[t]{2}{*}{ OR $(95 \% \mathrm{CI})$} \\
\hline & $\mathrm{C} / \mathrm{C}$ & $\mathrm{C} / \mathrm{A}$ & $\mathrm{A} / \mathrm{A}$ & $\chi^{2}$ & $\mathrm{P}$ & $\mathrm{C}$ & A & $\chi^{2}$ & $\mathrm{P}$ & \\
\hline Patients (K) & $0(0)$ & $4(0.03)$ & $116(0.97)$ & & 0.000 & $4(0.01)$ & $236(0.99)$ & & 0.000 & $0.15(0.052-0.447)$ \\
\hline Controls (K) & $0(0)$ & $24(0.20)$ & $96(0.80)$ & & & $24(0.10)$ & $216(0.90)$ & & & \\
\hline Patients (H) & $36(0.3)$ & $56(0.47)$ & $28(0.23)$ & 1.61 & 0.446 & $128(0.53)$ & $112(0.47)$ & 1.63 & 0.201 & $1.26(0.883-1.808)$ \\
\hline Controls (H) & $20(0.17)$ & $48(0.40)$ & $52(0.43)$ & & & $88(0.37)$ & $152(0.63)$ & & & \\
\hline
\end{tabular}

$\mathrm{P}<0.05$ indicates Statistical significance; $\mathrm{K}=$ Kazakh; $\mathrm{H}=$ Han. The parentheses represented the percentage of genotype and allele. 
Table 8. Chi-square test for the TNNT2 gene polymorphism.

\begin{tabular}{|c|c|c|c|c|c|c|c|c|c|c|}
\hline \multirow[t]{2}{*}{ Group } & \multicolumn{5}{|c|}{ rs3729547 genotype } & \multicolumn{4}{|c|}{ rs3729547 allele } & \multirow[t]{2}{*}{ OR $(95 \% \mathrm{CI})$} \\
\hline & $\mathrm{C} / \mathrm{C}$ & $\mathrm{C} / \mathrm{T}$ & $\mathrm{T} / \mathrm{T}$ & $\chi^{2}$ & $\mathrm{P}$ & $\mathrm{C}$ & $\mathrm{T}$ & $\chi^{2}$ & $\mathrm{P}$ & \\
\hline Patients (K) & $0(0)$ & $12(0.05)$ & $108(0.95)$ & & 0.000 & $12(0.05)$ & $228(0.95)$ & 4.94 & 0.039 & $0.45(0.222-0.923)$ \\
\hline Controls (K) & $0(0)$ & $25(0.21)$ & $95(0.79)$ & & & $25(0.17)$ & $215(0.82)$ & & & \\
\hline Patients $(\mathrm{H})$ & $24(0.20)$ & $52(0.43)$ & $44(0.37)$ & 4.60 & 0.101 & $100(0.42)$ & $140(0.58)$ & 2.43 & 0.142 & $0.75(0.524-1.077)$ \\
\hline Controls (H) & $26(0.22)$ & $65(0.54)$ & $29(0.24)$ & & & $117(0.48)$ & $123(0.52)$ & & & \\
\hline
\end{tabular}

$\mathrm{P}<0.05$ indicates Statistical significance; $\mathrm{K}=\mathrm{Kazakh} ; \mathrm{H}=\mathrm{Han}$. The parentheses represented the percentage of genotype and allele.

\section{DISCUSSION}

Single nucleotide polymorphisms (SNPs) are the most common type of genetic variation in the human genome, and many SNPs have been identified in patients with DCM. Two consecutive SNP screenings conducted in Europe showed that SNPs have close relationships with DCM (Kimura, 2011). TPM1 and TNNT2 gene mutation loci were detected in all Kazakh patients with IDCM. Such high mutation frequency was also common in the Han population. But after statistical analysis, the incidence of the Han nationality makes no sense. This is because Xinjiang is a remote area in China, and the diet, living habit, environment, and marital status of the Kazakh ethnic population are different from those of the Han ethnic population. This mutation might be transmitted to the next generation, furthermore, both genotype distributions and gene frequencies showed statistically significant differences in patients with DCM compared with the healthy Kazakh volunteers. The detection of SNP loci in the TPM1 and TNNT2 genes in both the Xinjiang Kazakh and Han population indicated that genetic factors are involved in the pathogenesis of disease (Table 4).

Previous studies have shown that the same genetic mutations can lead to HCM as well as causing DCM (Nanni et al., 2003), although the mechanisms remain unclear. Subsequent studies have pointed out that the same genetic mutations can lead to different appearances of HCM and DCM, which might be due to different functions of specific gene expression in these disorders (Debold et al., 2007; Robinson et al., 2007). For example, TNNT2 gene mutation can result in both decline and enhancement of myocardial contraction force in patients with DCM and HCM, respectively (Kamisago et al., 2000; Mirza et al., 2005). However, variability in clinical features within DCM induced by the same mutation loci apart from that which can be explained by genetic, environmental, and other factors has also been observed.

In our study, the gene mutation detection rates in Xinjiang Han patients with IDCM were $76 \%$ (TPM1) and 80\% (TNNT2). However, their genotype distributions and gene frequencies were not statistically significantly different compared with those of healthy Han volunteers, suggesting that although genetic factors play a major role in this disorder, external factors were also involved in its occurrence.

Abnormalities in cytoskeletons connecting the sarcomere and sarcolemma are mainly responsible for the pathogenesis of DCM. The fracture of this cytoskeleton can cause heart cavity expansion and decreased heart pump function. TPM1 encodes the sarcomere myofilament, is located on human chromosome 15q22.2, and contains 9 exons. The TPM1 gene product consists of two parallel polypeptide chains twisted into a spiral molecule and its function is to interact, strengthen, and stabilize the actin filament (Lakdawala et al., 2010). TPM1 combines 
with actin filament and troponin $\mathrm{T}$, their binding loci are composed of troponin complexes, which are sensitive to ionized calcium $\left(\mathrm{Ca}^{2+}\right)$. Therefore, increasing the $\mathrm{Ca}^{2+}$ levels promotes the movement of the tropomyosin-troponin complexes thereby prompting muscle contraction.

Three elements of the troponin complexes such as troponin T, joint troponin $\mathrm{C}$, and I comprise myocardial troponin, which can combine with myosin and adjust myocardial contraction and relaxation, and can coordinate with $\mathrm{Ca}^{2+}$ concentration-dependent adenine triphosphatase activity in the muscle fiber (Richard et al., 2003). Recent studies have shown that cardiac troponin not only maintains the structural integrity of the troponin complexes, but also affects the contraction force of the sarcomere components and myocardium (Sehnert et al., 2002). TNNT2 gene mutation not only affects the combination of the tropomyosin with troponin $\mathrm{T}$, and actin, but also changes the combination of the troponins C, I, and T (Mirza et al., 2005).

LMNA is the most commonly identified pathogenic gene in DCM. It is located on chromosome lq21.2-q21.3, and has a total genomic sequence length of $56.7 \mathrm{~kb}$ including a coding of approximately $24 \mathrm{~kb}$, which contains 12 exons (Wydner et al., 1996). LMNA geneencoded protein products include lamin $\mathrm{A}$ and $\mathrm{C}$, which are important components of the nucleus fiber layer and are the main cytoskeleton protein for maintaining normal nuclear membrane morphology. It has been confirmed that the first symptom of DCM induced by LMNA gene mutation is severe arrhythmia, whereas decreased heart function often appears late but progresses quickly in these patients (Bécane et al., 2000). However, in this study, most patients with IDCM were diagnosed with decreased ventricular function and heart ultrasonic LVEF was considered as an evaluation criterion. As these characteristics are inconsistent with the presentation following LMNA mutation, no LMNA gene mutations were detected.

The LVEDD and LVEF differences between the patients with IDCM and the healthy volunteers were statistically significant, which indicated that the genetic mutations might influence the expression of sarcomere proteins and the connection of tropomyosin with actin and troponin thus impacting myocardial cytoskeleton structure and inducing decreased myocardial systolic ability, ejection fraction, and systolic function leading to expanded ventricular cavity that ultimately results in the onset of DCM. As gene sequencing can speed up and simplify the diagnosis of disease and can inform medical treatment and health care in patients at the genome level, the risk of DCM in FDCM family members should be evaluated. Studies have shown that the genetic testing is beneficial for preventing the occurrence of patients with decreased cardiac function (evaluated by echocardiogram LVEF) (The SOLVD Investigators, 1992). Therefore, early diagnosis could prevent disease in patients with asymptomatic IDCM as well.

The TPM1 and TNNT2 genes might be susceptibility loci for patients with IDCM of Kazakh descent in Xinjiang. The results of this study results have suggested that the sarcomere gene mutation frequency in patients with IDCM was significantly higher than previously reported (Robinson et al., 2007). Familial transmission suggests that at least one kind of genetic mutation affects the onset of DCM. Therefore, it is necessary to carry out comprehensive gene sequencing of the patient, which might promote early diagnosis of the disease and enable timely treatment and prevention.

\section{ACKNOWLEDGMENTS}

We thank the Kazakh families, patients, volunteers, and the referring physicians for their participation in the Familial Dilated Cardiomyopathy Research Program, without whom 
these studies would not have been possible. Research supported by the National Natural Science Foundation of China (\#81260069).

\title{
Conflicts of interest
}

\author{
The authors declare no conflict of interest.
}

\section{REFERENCES}

Anonymous (1992). Effect of enalapril on mortality and the development of heart failure in asymptomatic patients with reduced left ventricular ejection fractions. N. Engl. J. Med. 327: 685-691.

Bécane HM, Bonne G, Varnous S, Muchir A, et al. (2000). High incidence of sudden death with conduction system and myocardial disease due to lamins A and C gene mutation. Pacing Clin. Electrophysiol. 23: 1661-1666.

Burkett EL and Hershberger RE (2005). Clinical and genetic issues in familial dilated cardiomyopathy. J. Am. Coll. Cardiol. 45: 969-981.

Debold EP, Schmitt JP, Patlak JB, Beck SE, et al. (2007). Hypertrophic and dilated cardiomyopathy mutations differentially affect the molecular force generation of mouse alpha-cardiac myosin in the laser trap assay. Am. J. Physiol. Heart Circ. Physiol. 293: H284-H291.

Fatkin D, Otway R and Richmond Z (2010). Genetics of dilated cardiomyopathy. Heart Fail. Clin. 6: 129-140.

Fatkin D and Members of the CSANZ Cardiac Genetic Diseases Council Writing Group (2011). Guidelines for the diagnosis and management of familial dilated cardiomyopathy. Heart Lung Circ. 20: 691-693.

Hershberger RE, Lindenfeld J, Mestroni L, Seidman CE, et al. (2009). Genetic evaluation of cardiomyopathy - a Heart Failure Society of America practice guideline. J. Card. Fail. 15: 83-97.

Kamisago M, Sharma SD, DePalma SR, Solomon S, et al. (2000). Mutations in sarcomere protein genes as a cause of dilated cardiomyopathy. N. Engl. J. Med. 343: 1688-1696.

Kimura A (2011). Contribution of genetic factors to the pathogenesis of dilated cardiomyopathy: the cause of dilated cardiomyopathy: genetic or acquired? (genetic-side). Circ. J. 75: 1756-1765.

Kubo T, Kitaoka H, Okawa M, Matsumura Y, et al. (2005). Lifelong left ventricular remodeling of hypertrophic cardiomyopathy caused by a founder frameshift deletion mutation in the cardiac myosin-binding protein $\mathrm{C}$ gene among Japanese. J. Am. Coll. Cardiol. 46: 1737-1743.

Lakdawala NK, Dellefave L, Redwood CS, Sparks E, et al. (2010). Familial dilated cardiomyopathy caused by an alphatropomyosin mutation the distinctive natural history of sarcomeric dilated cardiomyopathy. J. Am. Coll. Cardiol. 55: 320-329.

Lakdawala NK, Funke BH, Baxter S, Cirino AL, et al. (2012). Genetic testing for dilated cardiomyopathy in clinical practice. J. Cardiac Fail. 18: 296-303.

Mestroni L, Rocco C, Gregori D, Sinagra G, et al. (1999). Familial dilated cardiomyopathy: evidence for genetic and phenotypic heterogeneity. Heart Muscle Disease Study Group. J. Am. Coll. Cardiol. 34: 181-190.

Mirza M, Marston S, Willott R, Ashley C, et al. (2005). Dilated cardiomyopathy mutations in three thin filament regulatory proteins result in a common functional phenotype. J. Biol. Chem. 280: 28498-28506.

Mogensen J, Murphy RT, Shaw T, Bahl A, et al. (2004). Severe disease expression of cardiac troponin C and T mutations in patients with idiopathic dilated cardiomyopathy. J. Am. Coll. Cardiol. 44: 2033-2040.

Møller DV, Andersen PS, Hedley P, Ersbøll MK, et al. (2009). The role of sarcomere gene mutations in patients with idiopathic dilated cardiomyopathy. Eur. J. Hum. Genet. 17: 1241-1249.

Nanni L, Pieroni M, Chimenti C, Simionati B, et al. (2003). Hypertrophic cardiomyopathy: two homozygous cases with "typical" hypertrophic cardiomyopathy and three new mutations in cases with progression to dilated cardiomyopathy. Biochem. Biophys. Res. Commun. 309: 391-398.

Richard P, Charron P, Carrier L, Ledeuil C, et al. (2003). Hypertrophic cardiomyopathy: distribution of disease genes, spectrum of mutations, and implications for a molecular diagnosis strategy. Circulation 107: 2227-2232.

Robinson P, Griffiths PJ, Watkins H and Redwood CS (2007). Dilated and hypertrophic cardiomyopathy mutations in troponin and alphatropomyosin have opposing effects on the calcium affinity of cardiac thin filaments. Circ. Res. 101: 1266-1273.

Schönberger J and Seidman CE (2001). Many roads lead to a broken heart: the genetics of dilated cardiomyopathy. Am. J. Hum. Genet. 69: 249-260.

Sehnert AJ, Huq A, Weinstein BM, Walker C, et al. (2002). Cardiac troponin T is essential in sarcomere assembly and 
cardiac contractility. Nat. Genet. 31: 106-110.

The SOLVD Investigators (1992). Effect of enalapril on mortality and the development of heart failure in asymptomatic patients with reduced left ventricular ejection fractions. N. Engl. J. Med. 327: 685-691.

Wydner KL, McNeil JA, Lin F, Worman HJ, et al. (1996). Chromosomal assignment of human nuclear envelope protein genes $L M N A, L M N B l$, and $L B R$ by fluorescence in situ hybridization. Genomics 32: 474-478. 